\title{
Energy gaps and a zero-field quantum Hall effect in graphene by strain engineering
}

\author{
F. Guinea ${ }^{1 \star}$, M. I. Katsnelson ${ }^{2}$ and A. K. Geim ${ }^{3 \star}$
}

\begin{abstract}
Among many remarkable qualities of graphene, its electronic properties attract particular interest owing to the chiral character of the charge carriers, which leads to such unusual phenomena as metallic conductivity in the limit of no carriers and the half-integer quantum Hall effect observable even at room temperature ${ }^{1-3}$. Because graphene is only one atom thick, it is also amenable to external influences, including mechanical deformation. The latter offers a tempting prospect of controlling graphene's properties by strain and, recently, several reports have examined graphene under uniaxial deformation ${ }^{4-8}$. Although the strain can induce additional Raman features ${ }^{7,8}$, no significant changes in graphene's band structure have been either observed or expected for realistic strains of up to $\sim 15 \%$ (refs $9-11$ ). Here we show that a designed strain aligned along three main crystallographic directions induces strong gauge fields ${ }^{12-14}$ that effectively act as a uniform magnetic field exceeding $10 \mathrm{~T}$. For a finite doping, the quantizing field results in an insulating bulk and a pair of countercirculating edge states, similar to the case of a topological insulator ${ }^{15-20}$. We suggest realistic ways of creating this quantum state and observing the pseudomagnetic quantum Hall effect. We also show that strained superlattices can be used to open significant energy gaps in graphene's electronic spectrum.
\end{abstract}

If a mechanical strain $\Delta$ varies smoothly on the scale of interatomic distances, it does not break the sublattice symmetry but rather deforms the Brillouin zone in such a way that the Dirac cones located in graphene at points $\mathrm{K}$ and $\mathrm{K}^{\prime}$ are shifted in the opposite directions ${ }^{2}$. This is reminiscent of the effect induced on charge carriers by magnetic field $B$ applied perpendicular to the graphene plane $e^{2,12-14}$. The strain-induced, pseudomagnetic field $B_{\mathrm{S}}$ or, more generally, gauge-field vector potential A has opposite signs for graphene's two valleys $K$ and $K^{\prime}$, which means that elastic deformations, unlike magnetic field, do not violate the time-reversal symmetry of a crystal as a whole ${ }^{12-14,21,22}$.

On the basis of this analogy between strain and magnetic field, we ask the following question. Is it possible to create such a distribution of strain that it results in a strong uniform pseudomagnetic field $B_{\mathrm{S}}$ and, accordingly, leads to a 'pseudo-quantum Hall effect (QHE)' observable in zero $B$ ? The previous attempts to engineer energy gaps by applying strain ${ }^{5-7}$ seem to suggest a negative answer. Indeed, the hexagonal symmetry of the graphene lattice generally implies a highly anisotropic distribution of $B_{S}$ (refs 21, 22). Therefore, the strain is expected to contribute primarily in the phenomena that do not average out in a random magnetic field, such as weak localization ${ }^{13,14}$. Furthermore, a strong gauge field implies the opening of energy gaps owing to Landau quantization, $\delta E \approx 400 \mathrm{~K} \sqrt{B}\left(>0.1 \mathrm{eV}\right.$ for $\left.B_{\mathrm{S}}=10 \mathrm{~T}\right)$, whereas no gaps were theoretically found for uniaxial strain as large as $\approx 25 \%$ (ref. 4 ). The only way to induce significant gaps known so far is to spatially confine carriers $(\delta E \approx 0.1 \mathrm{eV} \text { requires } 10 \text {-nm-wide ribbons })^{1,2}$. Contrary to these expectations, we have found that by applying stresses with triangular symmetry it is possible to generate a uniform quantizing $B_{\mathrm{S}}$ equivalent to tens of Tesla so that the corresponding gaps exceed $0.1 \mathrm{eV}$ and are observable at room temperature.

A two-dimensional strain field $u_{i j}(x, y)$ leads to a gauge field ${ }^{23,24}$

$$
\mathbf{A}=\frac{\beta}{a}\left(\begin{array}{c}
u_{x x}-u_{y y} \\
-2 u_{x y}
\end{array}\right)
$$

where $a$ is the lattice constant, $\beta=-\partial \ln t / \partial \ln a \approx 2$ and $t$ the nearest-neighbour hopping parameter, and the $x$-axis is chosen along a zigzag direction of the graphene lattice. In the following, we consider valley $\mathrm{K}$, unless stated otherwise. We can immediately see that $B_{\mathrm{S}}$ can be created only by non-uniform shear strain. Indeed, for dilation (isotropic strain), equation (1) leads to $\mathbf{A}=0$ and, for the uniform strain previously considered in refs $4-6$, to $\mathbf{A}=$ const, which also yields zero $B_{\mathrm{S}}$.

Using polar coordinates $(r, \theta)$, equation (1) can be rewritten as

$$
\begin{aligned}
A_{r}= & \frac{\beta}{a}\left[\left(\frac{\partial u_{r}}{\partial r}-\frac{u_{r}}{r}-\frac{1}{r} \frac{\partial u_{\theta}}{\partial \theta}\right) \cos 3 \theta\right. \\
& \left.+\left(-\frac{1}{r} \frac{\partial u_{r}}{\partial \theta}+\frac{u_{\theta}}{r}-\frac{\partial u_{\theta}}{\partial r}\right) \sin 3 \theta\right] \\
A_{\theta}= & \frac{\beta}{a}\left[\left(-\frac{\partial u_{\theta}}{\partial r}+\frac{u_{\theta}}{r}-\frac{1}{r} \frac{\partial u_{r}}{\partial \theta}\right) \cos 3 \theta\right. \\
& \left.+\left(\frac{1}{r} \frac{\partial u_{\theta}}{\partial \theta}+\frac{u_{r}}{r}-\frac{\partial u_{r}}{\partial r}\right) \sin 3 \theta\right]
\end{aligned}
$$

which yields the pseudomagnetic field

$$
B_{\mathrm{S}}=\frac{\partial A_{y}}{\partial x}-\frac{\partial A_{x}}{\partial y}=\frac{1}{r} \frac{\partial A_{r}}{\partial \theta}-\frac{\partial A_{\theta}}{\partial r}-\frac{A_{\theta}}{r}
$$

In the radial representation, it is easy to show that uniform $B_{\mathrm{S}}$ is achieved for the following displacements:

$$
u_{r}=c r^{2} \sin 3 \theta, \quad u_{\theta}=c r^{2} \cos 3 \theta
$$

where $c$ is a constant. The strain described by (2) and its crystallographic alignment are shown in Fig. 1a,b, respectively. This yields uniform $B_{\mathrm{S}}=8 \beta c / a$ (given in units $\hbar / e \equiv 1$ ). For a

\footnotetext{
${ }^{1}$ Instituto de Ciencia de Materiales de Madrid (CSIC), Sor Juana Inés de la Cruz 3, Madrid 28049, Spain, ${ }^{2}$ Institute for Molecules and Materials, Radboud University Nijmegen, Heijendaalseweg 135, 6525 AJ, Nijmegen, The Netherlands, ${ }^{3}$ Manchester Centre for Mesoscience and Nanotechnology, University of Manchester, M13 9PL, Manchester, UK. *e-mail: paco.guinea@icmm.csic.es; geim@man.ac.uk.
} 


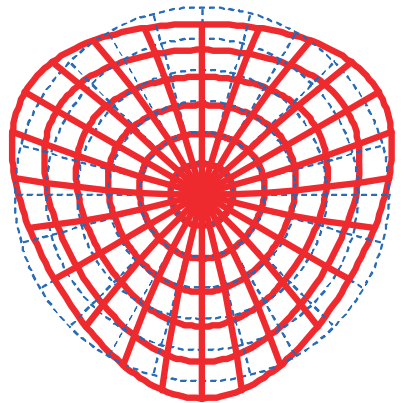

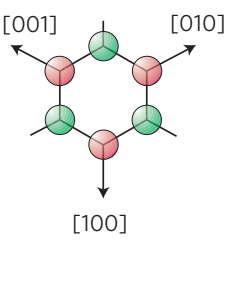

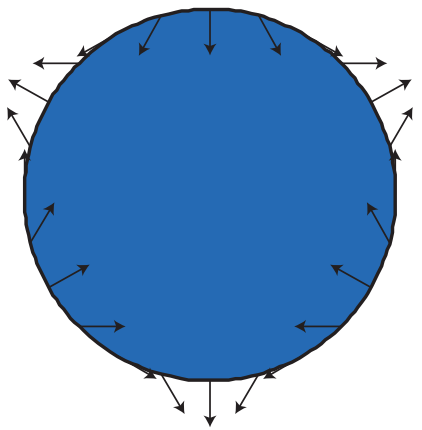

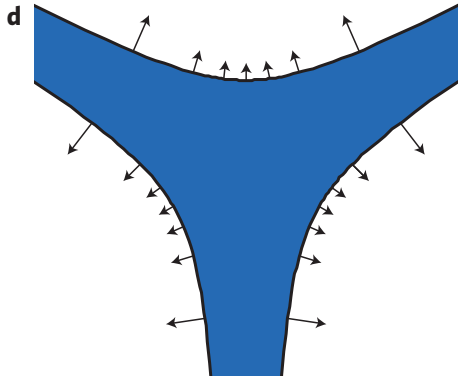

Figure 1 | Designed strain can generate a strictly uniform pseudomagnetic field in graphene. a, Distortion of a graphene disc which is required to generate uniform $B_{S}$. The original shape is shown in blue. $\mathbf{b}$, Orientation of the graphene crystal lattice with respect to the strain. Graphene is stretched or compressed along equivalent crystallographic directions $\langle 100\rangle$. Two graphene sublattices are shown in red and green. $\mathbf{c}$, Distribution of the forces applied at the disc's perimeter (arrows) that would create the strain required in $\mathbf{a}$. The uniform colour inside the disc indicates strictly uniform pseudomagnetic

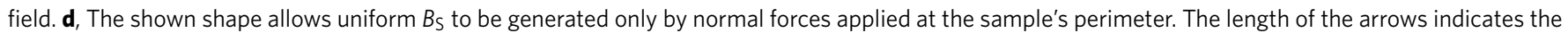
required local stress.

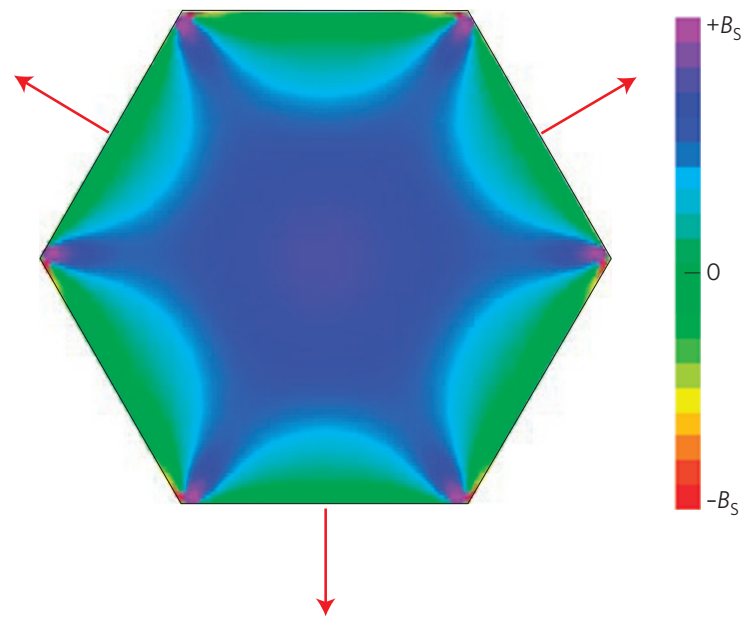

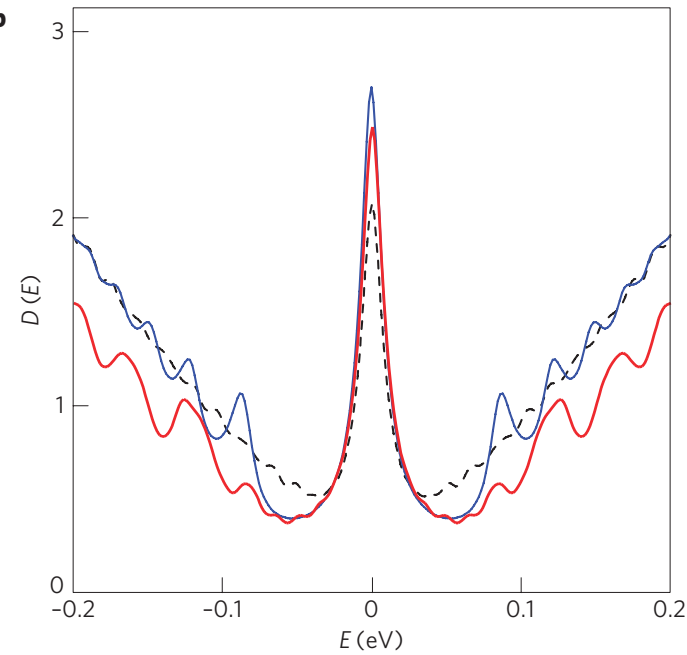

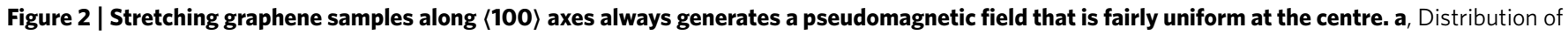
$B_{S}$ for a regular hexagon stretched by its three sides oriented perpendicular to $\langle 100\rangle$. Other examples are given in the Supplementary Information. b. Normalized density of states for the hexagon in a with $L=30 \mathrm{~nm}$ and $\Delta_{\mathrm{m}}=1 \%$. The black curve is for the case of no strain and no magnetic field. The

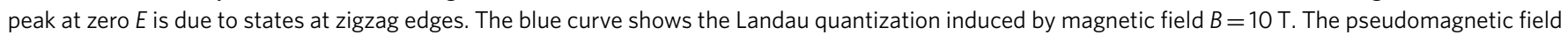
with $B_{\mathrm{S}} \approx 7 \mathrm{~T}$ near the hexagon's centre induces the quantization shown by the red curve. Comparison between the curves shows that the smearing of the

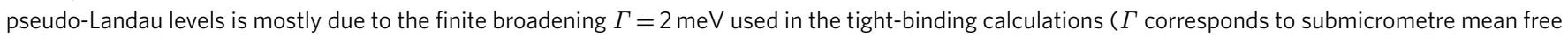
paths attainable in graphene devices). The inhomogeneous $B_{S}$ plays little role in the broadening of the first few pseudo-Landau levels (see Supplementary Fig. S4).

disc of diameter $D$, which experiences a maximum strain $\Delta_{\mathrm{m}}$ at its perimeter, we find $c=\Delta_{\mathrm{m}} / D$. For non-ambitious $\Delta_{\mathrm{m}}=10 \%$ and $D=100 \mathrm{~nm}$, we find $B_{\mathrm{S}} \approx 40 \mathrm{~T}$, the effective magnetic length $l_{\mathrm{B}}=\sqrt{a D / 8 \beta \Delta_{\mathrm{m}}} \approx 4 \mathrm{~nm}$ and the largest Landau gap of $\approx 0.25 \mathrm{eV}$. Note that distortions (2) are purely shear and do not result in any changes in the area of a unit cell, which means that there is no effective electrostatic potential generated by such strain ${ }^{23}$.

The lattice distortions in Fig. 1a can be induced by in-plane forces $\mathbf{F}$ applied only at the perimeter and, for the case of a disc, they are given simply by

$$
F_{x}(\theta) \propto \mu \sin (2 \theta), \quad F_{y}(\theta) \propto \mu \cos (2 \theta)
$$

where $\mu$ is the shear modulus. Figure 1c shows the required force pattern. It is difficult to create such strain experimentally because this involves tangential forces and both stretching and compression. To this end, we have solved an inverse problem to find out whether uniform $B_{\mathrm{S}}$ can be generated by normal forces only (Supplementary
Information, part I). There exists a unique solution for the shape of a graphene sample that enables this (see Fig. 1d).

A strong pseudomagnetic field should lead to Landau quantization and a QHE-like state. The latter is different from the standard QHE because $B_{\mathrm{S}}$ has opposite signs for charge carriers in valleys $\mathrm{K}$ and $\mathrm{K}^{\prime}$ and, therefore, generates edges states that circulate in opposite directions. The coexistence of gaps in the bulk and counterpropagating states at the boundaries without breaking the time-reversal symmetry is reminiscent of topological insulators ${ }^{15-20}$ and, in particular, the quantum valley Hall effect in 'gapped graphene ${ }^{30}$ and the quantum spin Hall effect induced by strain ${ }^{16}$. The latter theory has exploited the influence of three-dimensional strain on spin-orbit coupling in semiconductor heterostructures, which can lead to quasi-Landau quantization with opposite $B_{\mathrm{S}}$ acting on two spins rather than valleys. Weak spin-orbit coupling allows only tiny Landau gaps $<1 \mu \mathrm{eV}$ (ref. 16), which, to be observable, would require temperatures below $10 \mathrm{mK}$ and carrier mobilities higher than $10^{7} \mathrm{~cm}^{2} \mathrm{~V} \mathrm{~s}^{-1}$. Our approach exploits the 


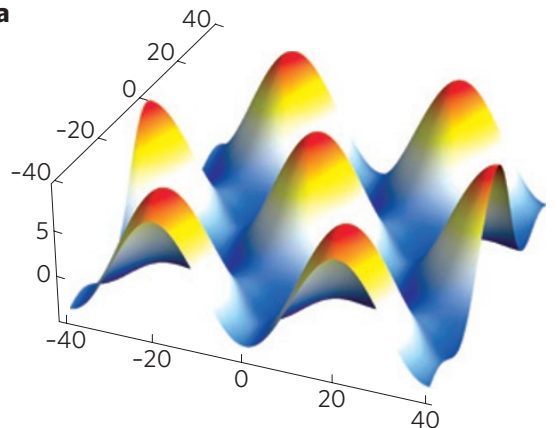

b

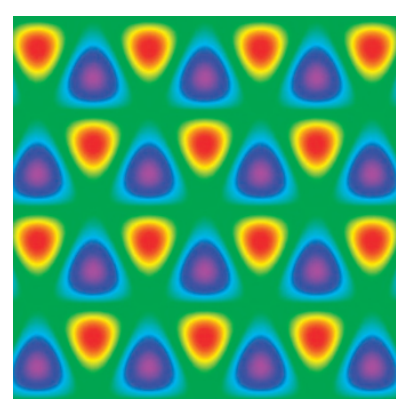

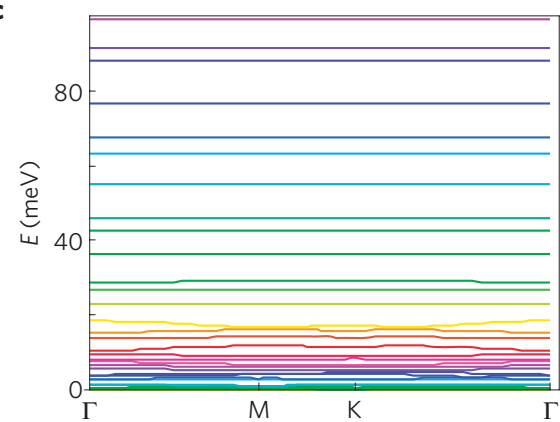

Figure 3 | Energy gaps can be opened in strained graphene superlattices. a, Strain with triangular symmetry can be created by depositing graphene on profiled surfaces (dimensions in the plot are given in nanometres). The corrugations shown in a result in the distribution of pseudomagnetic field plotted in $\mathbf{b}$. $B_{S}$ varies in the range $\pm 0.5 \mathrm{~T}$ (red to violet) with the periodicity half that in $\mathbf{a}$. c, Low-energy bands induced by the periodic strain. The bands are symmetric with respect to zero $E$.

unique strength of pseudospin-orbit coupling in graphene, which leads to $\delta E>0.1 \mathrm{eV}$ and makes the strain-induced Landau levels realistically observable.

The two cases shown in Fig. 1 prove that by using strain it is possible to generate a strong uniform $B_{\mathrm{S}}$ and observe the pseudoQHE. They also prove the general concept that if the strain is applied along all three $\langle 100\rangle$ crystallographic directions to match graphene's symmetry this prevents the generated fields from changing sign. However, it is a difficult experimental task to generate such a complex distribution of forces as shown in Fig. 1. Below we develop the found concept further and show that the pseudo-QHE can be observed in geometries that are easier to realize, even though they do not provide a perfectly uniform $B_{S}$.

Let us consider a regular hexagon with side length $L$ and normal stresses applied evenly at its three non-adjacent sides and along $\langle 100\rangle$ axes (Fig. 2a). Our numerical solution for this elasticity problem shows that $B_{\mathrm{S}}$ has a predominant direction (positive for $\mathrm{K}$ and negative for $\mathrm{K}^{\prime}$ ) and is fairly uniform close to the hexagon's centre. Assuming $L=100 \mathrm{~nm}$ and $\Delta_{\mathrm{m}}=10 \%$, we find for Fig. 2a that $B_{\mathrm{S}}$ varies in the range $\pm 22 \mathrm{~T}$ but is $\approx 20$ T over most of the hexagon's central area. For other $L$ and $\Delta$, we can rescale the plotted values of $B_{\mathrm{S}}$ by using the expression $B_{\mathrm{S}} \propto \Delta_{\mathrm{m}} / L$. We have also examined other geometries and always found a nearly uniform distribution of $B_{\mathrm{S}}$ near the sample's centre (see Supplementary Information).

To verify that the non-uniform $B_{S}$ in Fig. 2 a leads to welldefined Landau quantization, we have calculated the resulting density of states $D(E)$. To this end, we have used the scaling properties of the Dirac equation, which allows us to extrapolate the low-energy spectrum of small lattices to larger systems. The scaling approach is unfortunately limited to sizes $L \approx 30 \mathrm{~nm}$ (Supplementary Information, part V). Figure $2 \mathrm{~b}$ plots our results for $\Delta_{\mathrm{m}}=1 \%\left(B_{\mathrm{S}} \approx 7 \mathrm{~T}\right.$ at the hexagon centre) and compares them for the case of the same hexagon in $B=0$ and $10 \mathrm{~T}$ but without strain. In the absence of strain or $B$, the peak at zero $E$ is due to the states localized at the edges ${ }^{2}$. This peak increases with increasing strain, and its development is better seen in $D(E)$ calculated at the centre of the hexagon (Supplementary Fig. S5). We can also see that both non-uniform $B_{\mathrm{S}}$ and uniform $B$ generate Landau levels, and the qualities of the induced quantization are fairly similar. In Fig. 2b and Supplementary Fig. S5, the width of the zero- $E$ peak and the remnant density of states between zero and adjacent peaks are determined by the finite broadening $(\sim 2 \mathrm{meV})$ introduced in the calculation of Green's function, whereas the next two levels are slightly broadened by non-uniform $B_{S}$. In general, the influence of the inhomogeneity in $B_{\mathrm{S}}$ on the zero level should be minimal because field inhomogeneity does not lead to broadening of this level ${ }^{25}$. We emphasize that the smearing of the Landau levels in Fig. 2b is mostly due to the small sample size used in calculations and, for micrometre-sized hexagons, the first few pseudo-Landau levels should be well resolved in experiment (Supplementary Fig. S4).

To create the required strain, we can generally think of exploiting the difference in thermal expansion of graphene and a substrate ${ }^{11}$ and apply temperature gradients along $\langle 100\rangle$ axes. For the case of quasi-uniform $B_{\mathrm{S}}$, there are many more options available, including the use of suspended samples and profiled substrates. For example, a graphene hexagon can be suspended by three metallic contacts attached to its sides, similar to the technique used to study suspended graphene $e^{26,27}$, and the strain can then be controlled by gate voltage. Alternatively, a quasi-uniform $B_{\mathrm{S}}$ can be created by depositing graphene over triangular trenches (Supplementary Information).

To probe the pseudo-Landau quantization, we can use optical techniques, for example Raman spectroscopy, which should reveal extra resonances induced by $B_{S}$ (ref. 28). This technique should allow detection of pseudomagnetic field locally, within submicrometre areas. We can also use transport measurements in both standard and Corbino-disc geometries. In the former case, the counterpropagating edge states imply that contributions from two valleys cancel each other and no Hall signal is generated $\left(\rho_{x y}=0\right)$ (refs 15-20). At the same time, the edge transport can lead to longitudinal resistivity $\rho_{x x}=h / 4 e^{2} N$ where $N$ is the number of spin-degenerate Landau levels at the Fermi energy. This non-zero quantized $\rho_{x x}$ has the same origin as in so-called dissipative QHE, where two edge states with opposite spins propagate in opposite directions ${ }^{29}$. In spin-based topological insulators, the edge transport is protected by slow spin-flip rates ${ }^{15,16,29}$. In our case, atomic-scale disorder at the edges is likely to mix the countercirculating states on a submicrometre scale (Supplementary Information, section VI). Therefore, instead of quantization in $\rho_{x x}$ we may expect highly resistive metallic edge states, similar to the case discussed in ref. 29. The suppression of the edge-state ballistic transport does not affect the pseudo-Landau quantization in graphene's interior, where intervalley scattering is very weak ${ }^{13,30}$ and should not cause extra level broadening. Highly resistive edges should in fact make it easier to probe pseudo-Landau gaps in the bulk. In the Corbino geometry, the edge-state mixing is irrelevant, and we expect two-probe $\rho_{x x}$ to be a periodic function of gate voltage and show an insulating behaviour between pseudo-Landau levels. Furthermore, the outer contact can be used to cover perimeter regions, in which $B_{\mathrm{S}}$ is non-uniform. This should improve the quality of quantization.

Finally, we point out that the developed concept can be used to create gaps in bulk graphene. Imagine a macroscopic graphene sheet deposited on top of a corrugated surface with a triangular landscape (Fig. 3a). In the following calculations, we have fixed the graphene sheet at the landscape's extrema and enabled the resulting in-plane 
displacements to $\operatorname{relax}^{21,22}$ (at the nanoscale, graphene should then be kept in place by van der Waals forces). The resulting pseudomagnetic superlattice is plotted in Fig. $3 \mathrm{~b}$ whereas Fig. $3 \mathrm{c}$ shows the resulting energy spectrum. Close to zero $E$, there is a continuous band of electronic states, in agreement with the fact that the zero level is insensitive to the field's inhomogeneity ${ }^{25}$. At higher $E$, there are multiple gaps with $\delta E>100 \mathrm{~K}$. The relatively small gaps are due to the weak shear strain induced in this geometry $\left(\Delta_{\mathrm{m}}<0.1 \%\right)$. By improving the design of strained superlattices, it must be possible to achieve much larger gaps. We believe that the suggested strategies to observe the pseudo-Landau gaps and QHE are completely attainable and will be realized sooner rather than later.

Received 26 May 2009; accepted 1 September 2009; published online 27 September 2009

\section{References}

1. Geim, A. K. \& Novoselov, K. S. The rise of graphene. Nature Mater. 6, 183-191 (2007).

2. Castro Neto, A. H., Guinea, F., Peres, N. M. R., Novoselov, K. S. \& Geim, A. K. The electronic properties of graphene. Rev. Mod. Phys. 81, 109-162 (2009).

3. Novoselov, K. S. et al. Room temperature quantum Hall effect in graphene. Science 315, 1379 (2007).

4. Pereira, V. M., Castro Neto, A. H. \& Peres, N. M. R. Tight-binding approach to uniaxial strain in graphene. Phys. Rev. B 80, 045401 (2009).

5. Ni, Z. et al. Uniaxial strain on graphene: Raman spectroscopy study and band-gap opening. ACS Nano 3, 483 (2009).

6. Farjam, M. \& Rafii-Tabar, H. Comment on band structure engineering of graphene by strain: First-principles calculations. Preprint at $<$ http://arxiv.org/abs/0903.1702> (2009).

7. Mohiuddin, T. M. G. et al. Uniaxial strain in graphene by Raman spectroscopy: G peak splitting, Gruneisen parameters and sample orientation. Phys. Rev. B 79, 205433 (2009).

8. Huang, M. et al. Raman spectroscopy of graphene under uniaxial stress: Phonon softening and determination of the crystallographic orientation. Proc. Natl Acad. Sci. USA 106, 7304-7308 (2009).

9. Lee, C., Wei, X., Kysar, J. \& Hone, J. Measurement of the elastic properties and intrinsic strength of monolayer graphene. Science 321, 385-388 (2008).

10. Kim, K. S. et al. Large-scale pattern growth of graphene films for stretchable transparent electrodes. Nature 457, 706-710 (2009).

11. Bao, W. et al. Controlled ripple texturing of suspended graphene and ultrathin graphite membranes. Nature Nanotech. 4, 562-566 (2009)

12. Sasaki, K., Kawazoe, Y. \& Saito, R. Local energy gap in deformed carbon nanotubes. Prog. Theor. Phys. 113, 463-480 (2005).

13. Morozov, S. V. et al. Strong suppression of weak localization in graphene. Phys. Rev. Lett. 97, 016801 (2006).

14. Morpurgo, A. F. \& Guinea, F. Intervalley scattering, long-range disorder, and effective time-reversal-symmetry breaking in graphene. Phys. Rev. Lett. 97, 196804 (2006)
15. Kane, C. L. \& Mele, E. J. Quantum spin Hall effect in graphene. Phys. Rev. Lett. 95, 226801 (2005).

16. Bernevig, B. A. \& Zhang, S. C. Quantum spin Hall effect. Phys. Rev. Lett. 96, $106802(2006)$

17. Moore, J. E. \& Balents, L. Topological invariants of time-reversal-invariant band structures. Phys. Rev. B 75, 121306 (2007).

18. Qi, X.-L., Hughes, T. L. \& Zhang, S.-C. Topological field theory of time-reversal invariant insulators. Phys. Rev. B 78, 195424 (2008).

19. Martin, I., Blanter, Ya. M. \& Morpurgo, A. F. Topological confinement in bilayer graphene. Phys. Rev. Lett. 100, 036804 (2008).

20. Semenoff, G. V., Semenoff, V. \& Zhou, F. Domain walls in gapped graphene. Phys. Rev. Lett. 101, 087204 (2008).

21. Guinea, F., Horovitz, B. \& Le Doussal, P. Gauge field induced by ripples in graphene. Phys. Rev. B 77, 205421 (2008).

22. Wehling, T. O., Balatsky, A. V., Tsvelik, A. M., Katsnelson, M. I. \& Lichtenstein, A. I. Midgap states in corrugated graphene: $A b$ initio calculations and effective field theory. Europhys. Lett. 84, 17003 (2008).

23. Suzuura, H. \& Ando, T. Phonons and electron-phonon scattering in carbon nanotubes. Phys. Rev. B 65, 235412 (2002).

24. Mañes, J. L. Symmetry based approach to electron-phonon interactions in graphene. Phys. Rev. B 76, 045430 (2007).

25. Giesbers, A. J. et al. Quantum-Hall activation gaps in graphene. Phys. Rev. Lett. 99, 206833 (2007)

26. Bolotin, K. I. et al. Ultrahigh electron mobility in suspended graphene. Solid State Commun. 146, 351-355 (2008).

27. Xu, D., Skachko, I., Barker, A. \& Andrei, E. Y. Approaching ballistic transport in suspended graphene. Nature Nano 3, 491-495 (2008).

28. García-Flores, A. F., Terashita, H., Granado, E. \& Kopelevich, Y. Landau levels in bulk graphite by Raman spectroscopy. Phys. Rev. B 79, 113105 (2009).

29. Abanin, D. A. et al. Dissipative quantum Hall effect in graphene near the Dirac point. Phys. Rev. Lett. 98, 196806 (2007).

30. Tikhonenko, F. V., Horsell, D. W., Gorbachev, R. V. \& Savchenko, A. K. Weak localization in graphene flakes. Phys. Rev. Lett. 100, 056802 (2008).

\section{Acknowledgements}

This work was supported by the EPSRC (UK), FOM (The Netherlands), the Royal Society, Office of Naval Research and Air Force Office of Scientific Research. F.G. also acknowledges support by MICINN (Spain) through grants FIS2008-00124 and CONSOLIDER CSD2007-00010, and by the Comunidad de Madrid, through CITECNOMIK.

\section{Author contributions}

All authors contributed extensively to various aspects of the work presented in this paper.

\section{Additional information}

The authors declare no competing financial interests. Supplementary information accompanies this paper on www.nature.com/naturephysics. Reprints and permissions information is available online at http://npg.nature.com/reprintsandpermissions. Correspondence and requests for materials should be addressed to F.G. or A.K.G. 